

\section{REVISTA \\ TEORÍA Y PRÁCTICA \\ DE LA \\ ARQUEOLOGÍA HISTÓRICA LATINOAMERICANA}

ISSN: 2250-866X (impreso) | ISSN: 2591-2801 (en línea)

AÑo VI, Volumen 6, Verano DE 2017

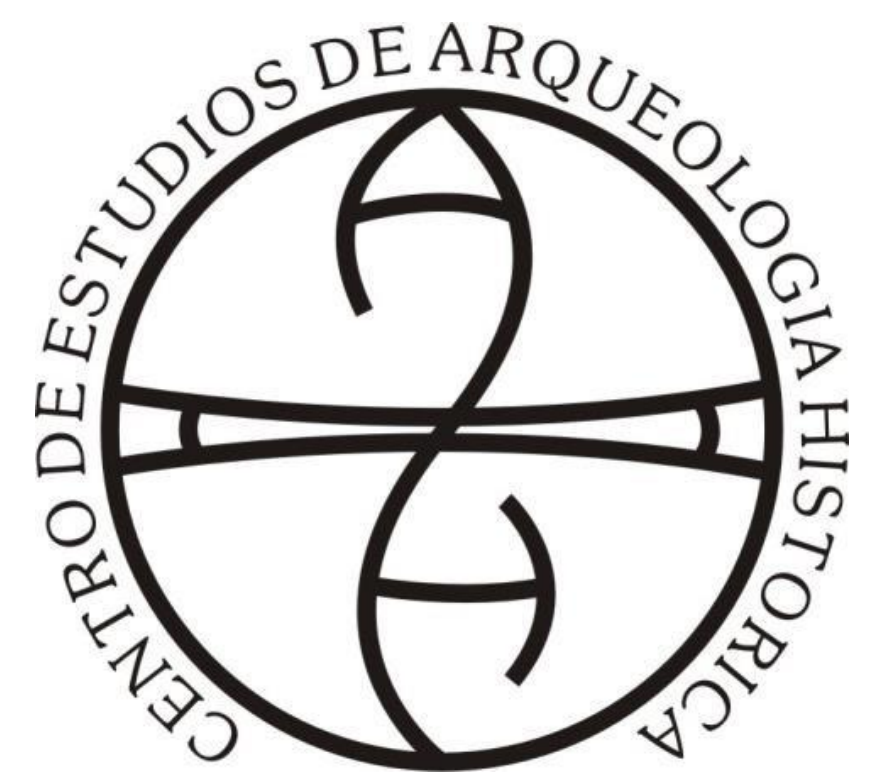

Centro de Estudios de Arqueología Histórica

FACULTAD DE HUMANIDADES Y ARTES | UNIVERSIDAD NACIONAL DE ROSARIO 

PARTICIPA EN LA RED DE ESTUDIOS INTEGRADOS SOBRE LOS PAISAJES SUDAMERICANOS

(Universidad Nacional de Rosario, Universidad Nacional de Río Cuarto,

Universidad Nacional de San Juan, Universidad de la República, Universidad Nacional de Trujillo)

AUTORIDADES DE LA UNIVERSIDAD NACIONAL DE ROSARIO

Rector: Dr. Arq. Héctor Floriani

Vicerrector: Lic. Fabián Bicciré

Secretario general: Lic. Mariano Balla

Secretario académico: Dr. Carlos A. Hernández

\author{
AUTORIDADES DE LA FACULTAD DE HUMANIDADES Y ARTES \\ Decano: Prof. José Goity \\ Vicedecano: Prof. Marta Varela \\ Secretaria Académica: Dra. Liliana Pérez
}

AUTORIDADES DEL CENTRO DE ARQUEOLOGÍA HISTÓRICA

Directora: Dra. Ana María Rocchietti

Secretaria: Prof. Nélida De Grandis

Prosecretaria: Lic. Marianela Biscaldi

Revista del Centro de Estudios de Arqueología Histórica

Teoría y Práctica de la Arqueología Histórica Latinoamericana Actas del Sexto Simposio de Arqueología Histórica Latinoamericana

Presidente del Simposio: Licenciada Mónica Valentini
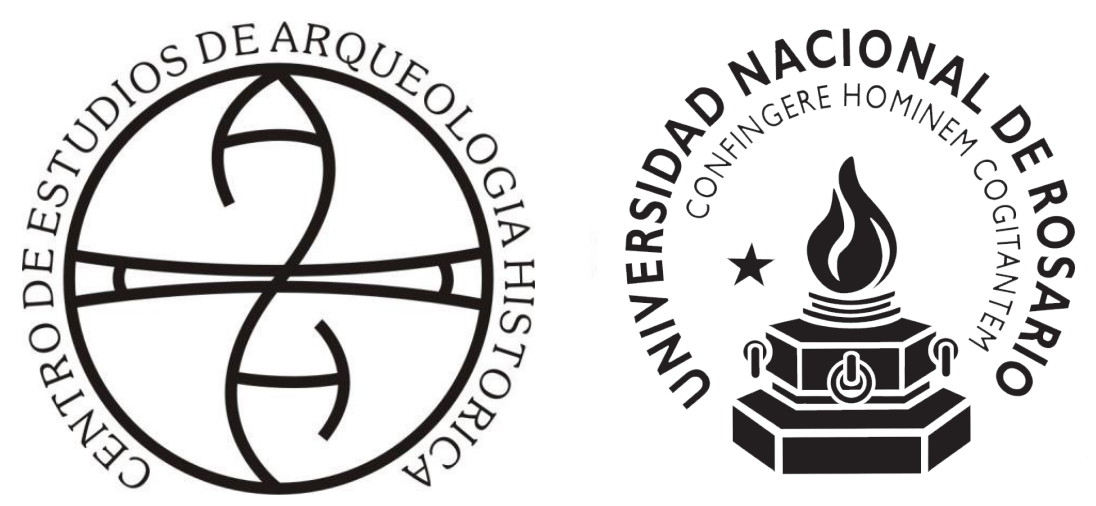


\section{Directoras Editoras}

Ana María Rocchietti y Nélida De Grandis

\section{Secretaria}

Irene Dosztal

\section{Comité Editor}

Ana María Rocchietti, Mónica P. Valentini

Nélida De Grandis, Marianela Biscaldi

Irene Dosztal, María Victoria Roca,

Fátima Solomita Banfi

\section{Comité Científico del V Simposio}

Dra. Nidia Areces

Dr. Raúl Bolmaro

Dr. Luis María Calvo

Lic. Juan Castañeda Murga

Lic. Carlos Ceruti

Dr. Juan Bautista Leoni

Dra. Eugenia Néspolo

Lic. Ruth Adela Poujade

Ing. Adrián Pifferetti

Dr. Mariano Ramos

Dr. Daniel Schávelzon

Dra. Marcela Tamagnini

Dra. Alicia Tapia

\section{Comité Científico}

Dr. Raúl Bolmaro (Universidad Nacional de Rosario)

Dr. Luis María Calvo (Museo Etnográfico de Santa Fe y Parque Arqueológico de Santa Fe La Vieja)

Lic. Carlos Ceruti (CONICET)

Dra. Dora de Grinberg (Facultad de Ingeniería, UNAM)

Dra. Eugenia Néspolo (Universidad Nacional de Luján)

Ing. Tulio Palacios (Comisión Nacional de Energía Atómica)

Lic. Ruth Poujade (Programa Misiones

Jesuíticas - Provincia de Misiones)

Dr. Mariano Ramos (Universidad Nacional de Luján)

Dra. Ana María Rocchietti (Universidad

Nacional de Rosario)
Dr. Daniel Schávelzon (Centro de

Arqueología Urbana - Universidad de

Buenos Aires)

Dra. Marcela Tamagnini (Universidad

Nacional de Río Cuarto)

Dra. Alicia Tapia (Universidad de Buenos

Aires)

Dr. Arno Álvarez Kern (Centro Nacional de

Pesquisas - Brasil)

Dra. Noemí Walsöe de Reca (CONICET)

Lic. Mónica Valentini (Universidad

Nacional de Rosario)

\section{Evaluaron este Volumen}

Horacio Chiavazza, Mónica Valentini,

Nélida De Grandis, Mariano Ramos, Juan

Bautista Leoni, Ana María Rocchietti, Javier García Cano, Flavio Ribero

\section{Diseño y diagramación}

Eugenia Reboiro

(eugenia.reboiro@gmail.com)

\section{Curaduría}

Irene Dosztal y Ana María Rocchietti

Foto de tapa: botellas de vidrio del texto de Orsini y Padula.

\section{Propietario responsable:}

Universidad Nacional de Rosario

Facultad de Humanidades y Artes,

Centro de Estudios de Arqueología

Histórica.

Entre Ríos 758. Rosario, Provincia de Santa

Fe (2000). Argentina.

Telf.: +54 (0341) 4802670

E-mail: ceahunr@gmail.com

Decreto Ley 6422/57 de Publicaciones Periódicas 


\section{Índice}

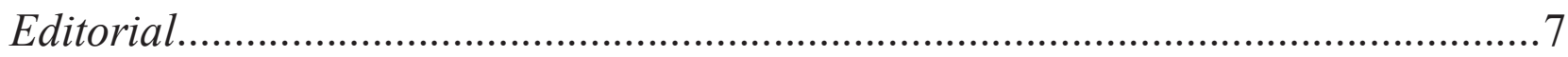

Experimentación, transferencia tecnológica y conservadurismo: notas sobre la introducción del aforro de cobre en los barcos de madera del siglo XVIII......

Nicolás C. Ciarlo

Arqueología social latinoamericana y los desafios de la arqueología urbana.

Ana María Rocchietti

"No todo fue hispánico en la casa de los Larreta". Intervención arqueológica en el jardín del Museo Larreta.

Ricardo Orsini y Horacio Padula

La construcción con ladrillo asentado en barro. El caso de la ciudad de

Rosario

Adrián A. Pifferetti

“Mientras las gárgolas nos miran”. Un sitio arqueológico urbano ¿y religioso?

en la ciudad de Luján. Provincia de Buenos Aires.

Mariano Ramos, Alejandra Raies y Matías Warr

Asentamientos europeos en la cuenca del Río de la Plata: primeras

aproximaciones a Corpus Christi.

Fernando Balducci, Julieta Sartori, Paula Galligani, Fernando Roggero, Macarena Riberi y Abril Castagnola

"De lugares y objetos". La visibilización del pasado afro en el norte de Argentina. Caso de estudio: la ciudad colonial de San Miguel de Tucumán.... 79

Luciana Chávez

Teledetección espacial aplicada a la búsqueda de restos de la Estancia San Miguel del Carcarañá.

Fabián Bognanni, Mónica P. Valentini, Mariano Darigo, Matías Warr y María Lara Moschetoni 
Rescate arqueológico. Hotel Pensión Europea. Posadas.

Misiones. Argentina.

Marianela Biscaldi y Lorena Salvatelli

El Fuerte Independencia revive tres siglos después en medio de un debate sobre sus túneles

Julio Fabián Merlo y María del Carmen Langiano

Estudio de muestras metálicas de vado de Las tres cascadas, Río Cuarto, Córdoba

Adrián A. Pifferetti

Clasificación de botones Prosser y su potencial como indicador

cronológico. Arqueología urbana de Rosario (sitio La Basurita)

María Fernanda Bruzzoni y Sandra Escudero

Una casa del siglo XX y un fuerte del siglo XVI. Arqueología

histórica en Cobos.

Jorge Esteban Cabral Ortiz y Rosana Jimena Flores

Un fechado intrigante

Mario Silveira y Mónica Patricia Valentini

Criterios adoptados para la conservación de los restos de estructura naval del Pecio Zencity de Puerto Madero (Buenos Aires, Argentina): ¿¿RE-ENTERRAR PARA CONSERVAR?!.

Eva Tavella 


\title{
ESTUDIO DE MUESTRAS METÁliCAS DE VADO de Las tres cascadas, Río Cuarto, Córdoba
}

\author{
Adrián A. Pifferetti *
}

\begin{abstract}
Resumen
Se ha estudiado un lote de materiales metálicos provenientes de trabajos arqueológicos en el sitio vado de las Tres Cascadas, zona serrana de Rio Cuarto. Se trata una tijera despabiladora, una grapa de acero de uso en vestimenta, una pieza cilíndrica de aleación de cobre de algún elemento de iluminación a altura de mecha regulable, un trozo de varilla rectangular de acero usado como jalón y dos cuchillos de un juego de fabricación industrializada en serie. El análisis del conjunto, sin descartar que pueda haber alguna pieza un poco más antigua como la tijera despabiladora, nos lleva a ubicar cronológicamente al conjunto a los últimos años del siglo XIX y, más posiblemente a principios del XX.
\end{abstract}

Palabras clave: arqueometalurgia, piezas metálicas, tecnología, análisis metalográfico, caracterización.

\begin{abstract}
It has been studied a lot of metal materials from archaeological work at the ford site of the Three Waterfalls, mountainous area of Rio Cuarto. One despabilator scissors, a staple steel use in clothing, a cylindrical piece of copper alloy of any element lighting height adjustable wick, a piece of rectangular steel rod used as milestone and two knives of a game is industrialized mass production. Analysis of all, without ruling out that there may be some slightly older piece like the despabiladora scissors, leads us to chronologically place the set in the late nineteenth century and, possibly early twentieth centuries.
\end{abstract}

Keywords: archaeometallurgy, metal parts, technology, metallographic analysis, characterization.

\section{Introducción}

Se han estudiado una serie de piezas metálicas provenientes de trabajos arqueológicos en el sitio Vado de las Tres Cascadas, zona serrana de Río Cuarto. Se trata de un sitio que muestra algunos restos de una vivienda en un sitio relativamente aislado. Se trata una tijera despabiladora, una grapa de acero de uso en vestimenta, una pieza cilíndrica de aleación de cobre, un trozo de planchuela o varilla rectangular de acero usado como jalón o puntal, ya que un extremo muestra haber sido deforma-

\footnotetext{
* Centro de Estudio de Materiales y Tecnologías, Universidad Tecnológica Nacional, Facultad Regional Rosario. Centro de Estudios de Arqueología Histórica. Universidad Nacional de Rosario. apiffere@gmail.com
} 
do por martillado y dos hojas de cuchillo similares Nuestro trabajo consistió en la limpieza, medición y análisis de las mismas para poner de manifiesto todas sus características y detalles para determinar la forma de la pieza, el proceso de fabricación y, dentro de lo posible, su ubicación cronológica en el proceso de población de la zona.

\section{Metodología y técnicas de estudio}

Las piezas fueron medidas con calibres de precisión, todas las medidas de dan en mm, como es común en la práctica ingenieril. En las muestras 2 y 6 , que lo permitían se extrajeron muestras para realizar análisis macro y micrográficos, en la pieza cilíndrica de aleación no ferrosa se extrajo el pequeño perno y en la varilla de acero al carbono se cortó una zona de $5 \mathrm{~mm}$ en el extremo no martillado. La observación de las superficies se realizó mediante una lupa estereoscópica binocular con 10 y 20x y los análisis metalográficos utilizando un microcopio Olimpus de platina invertida con aumentos variables entre 100 y $800 x$. Las probetas metalográficas fueron montadas en resina acrílica y luego de una preparación superficial con papeles esmeriles bajo agua hasta granulometría 1000 , con un posterior pulido con alúmina, fueron atacadas químicamente, la de acero con solución alcohólica de ácido nítrico (Nital) al 2 \% y la de aleación de cobre con una solución acuosa de cloruro férrico al $5 \%$ en volumen.

\section{Descripción de las piezas}

Muestra 1 identificada como "Vado de las Tres Cascadas, Recinto 2, S2 W8, 0,20,025"

$15 / 7 / 2013$. Se trata de una tijera de acero sumamente oxidada de un largo conservado de 148 y unos ojos de los que sólo uno se ha conservado entero teniendo un largo de 33,6 y un ancho de 23 con un espesor de unos $3 \mathrm{~mm}$ y la sección parece redondeada. Parece haber perdido algunos milímetros el la punta y presenta lateralmente un sector semicircular de 23,5 x 44,5 la altura exterior de este sector es de 13,3 y la profundidad del vacío interior aunque presenta productos de corrosión y tierra muy adherida es de 6 .

Muestra 2 identificada como "Vado de las Tres Cascadas, Recinto 2, S2 W8, 020-0,25"

14/07/2013. Es una pieza cilíndrica de aleación de cobre, compuesta por tres o cuatro elementos, que termina en un extremo con una ligera conicidad. Está formada por un cuerpo cilíndrico hueco de unos 25,6 de lago (o tal vez 1 pulgada), de un diámetro de 14,3 y espesor de 0,5; con una ligera saliente en un extremo en que es abrazado por un aro hueco de perímetro moleteado. Este anillo abraza también a una pieza cónica hueca de una altura de diez, y un diámetro mayor de 15 y menor de 10,5 y el mismo espesor de 0,5. Interiormente corre otra pieza cilíndrica hueca de similar espesor y que sobresale unos $2,5 \mathrm{~mm}$ y presenta una rosca. El cilindro exterior presenta una ranura inclinada que termina hacia la parte cónica en cuatro muescas que donde podía insertarse un pequeño pernito circular de $1 \mathrm{~mm}$ de diámetro. En correspondencia de las muescas en el cilindro hay unos números márcados (Figura 1). 


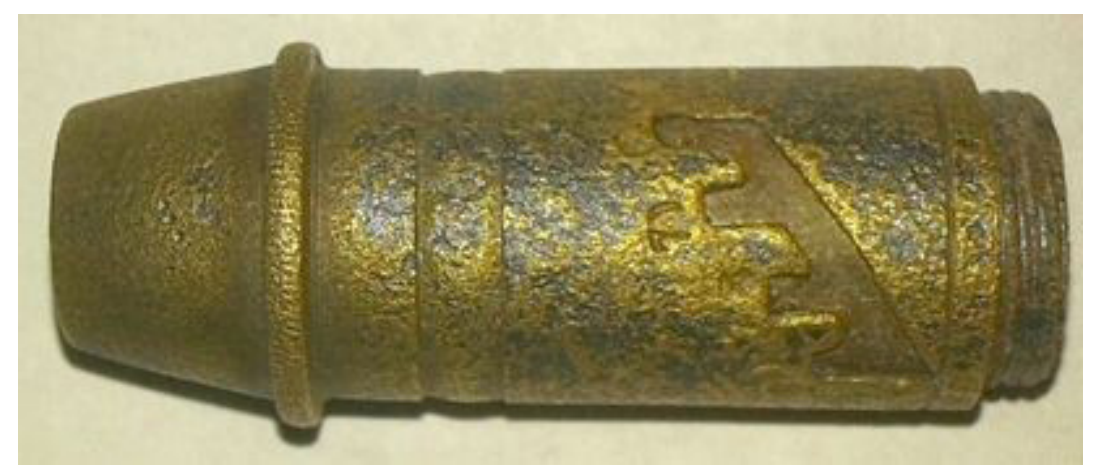

Figura 1. Muestra 2 Pieza compuesta de aleación de cobre

Muestra 3 identificada como "Paraje Vado de las Tres Cascadas, Alero 1, S8E2; P=1,93, A=0,28, $\mathrm{O}=0,48$ " 20/07/2013. Se trata de una pieza de aleación ferrosa muy corroída recubierta de productos de corrosión amarronados de distintas tonalidades. La hoja es triangular de 112,7 de lago x 111,6 conservados en la zona del filo x 27,5 de alto máximo siendo su espesor de 2,2 y termina en una sección ligeramente trapezoidal de 55,3 de lago x 15,4 en la zona del resalte y 20 en el extremo opuesto. El espesor de esta última sección es mayor, alrededor de 3. En la zona de unión de la hoja triangular con el área trapezoidal presenta un resalte bifacial rectangular de unos 11 x $7 \mathrm{~mm}$, hasta alcanzar un espesor de 9,1-9,2.

Muestra 4 identificada como "Vado de las Tres Cascadas Recinto 2 S2 W8 0,20-0,25 14/07/2013". Pieza similar a la $\mathrm{N}^{0} 3$, aunque la hoja triangular ha perdido la punta. El largo conservado es de 116 x 113,4 en la zona del filo x 25 de altura máxima, con un espesor fluctuante entre 1,9 y 2,4. La zona trapezoidal tiene 61,7 de largo x 14,2 x 20,6 en la terminación bien conservada y que se aprecia redondeada; su espesor es de unos 3,5. El resalte es de 11,2 x 7,2 alcanzando un espesor de 9,5 (Figura 2).

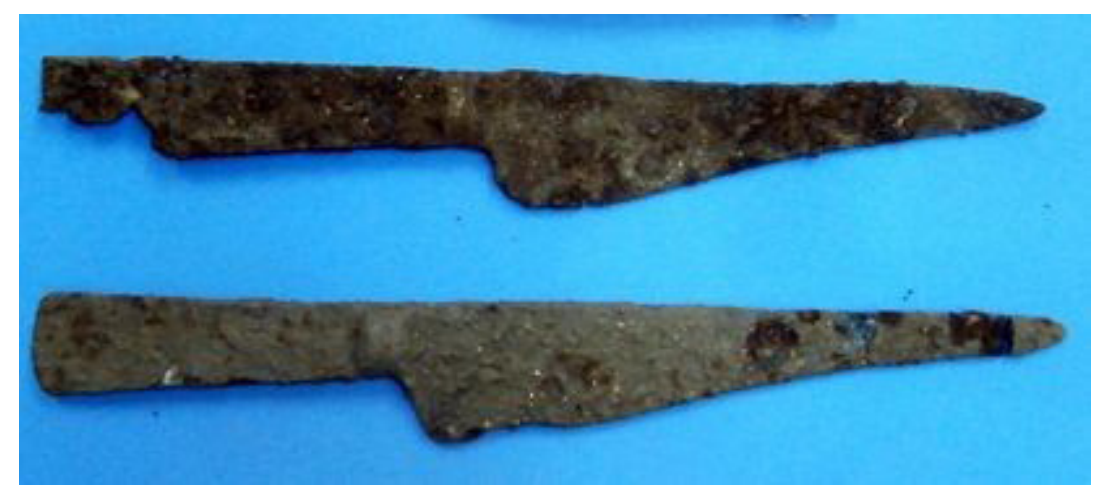

Figura 2. Cuchillos fabricados en serie

Muestra 5 identificada como "Paso del Vado Cascada, Alero 2, superficie, elemento de metal" 13/7/2013. SE trata de una pieza de aleación ferrosa menos corroída que las anteriores, de alambre redondo de 2,1 de diámetro, conformado para formar una presilla de fijación para coser en la ropa. Sus dimensiones son 23 de alto x 17 de ancho.

Muestra 6 identificada como "Vado de las Tres Cascadas, Recinto 2, S2 W8, 0,20-0,30"

15/07/2013. Trozo de planchuela de acero de $290 \mathrm{~mm}$ de largo y sección rectangular de 17,7 x 7 , los extremos lárgos son rectos mientras que los cortos son redondeados. Presenta una marcada corrosión superficial y un extremo muy deformado por martillado como si hubiera sido utilizado como jalón incado en el terreno. 


\section{Resultados}

La muestra 1 es sin ninguna duda una tijera despabiladora de las que se usaban para limpiar las mechas o pábilos de las velas fabricadas con grasas animales o vegetales (sebo) las que requerían la periódica eliminación de la mecha carbonizada y los restos de sebo que a ella se adherían para lograr una combustión eficiente. Es como una tijera común pero una de las hojas tiene un pequeño recipiente para recoger los restos de cera y mecha. Esta tijera despabiladora es similar a las de Santa Fe La Vieja (Pifferetti, 2004:57; Sanchez Beltran, 1993) aunque aquellas suelen ser de tamaño algo mayor. Las velas de parafina obtenida del petróleo se comenzaron a utilizar en la segunda mitad del siglo XIX y se generalizaron a fines de ese siglo (Fondazione Neri, 213).

La pieza compuesta 2 es parte de un quemador que permitía regular la altura de una mecha, y formó parte de algún tipo de farol de iluminación o calentador a combustible líquido (petróleo o querosén). Su uso se generalizó a partir de 1870 (Roldan, 1953; Guillman, 1886). El cuerpo cilíndrico exterior presenta una unión longitudinal por soldadura térmica.

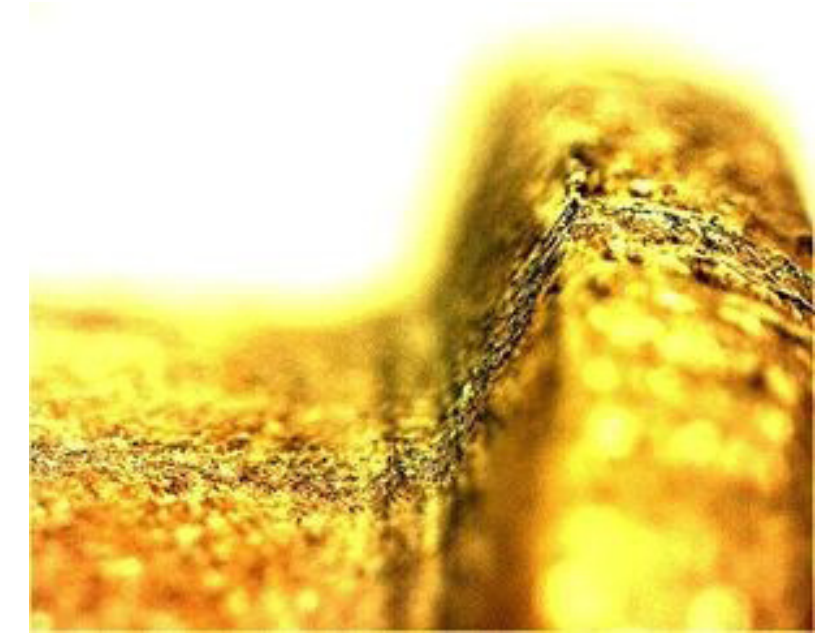

Figura 3. Pieza 2 de aleación de cobre. Zona de unión por soldadura.

Las piezas 3 y 4 son dos cuchillos que formaron parte de un juego de fabricación industrial en serie comprados en algún almacén de ramos generales o en un negocio urbano. Schavelzon (1991, 158) nos dice que la producción industrial de cubiertos se inicia "recién en 1840-1850"

La pieza 5 en una presilla o mitad hembra de un corchete, gancho o broche metálico de uso en vestimenta. El corchete es un broche metálico conformado por dos elementos, el gancho y la presilla (o macho y hembra) y permite la unión por tracción mecánica entre dos partes de una prenda. Su tamaño indica que se usó en un pantalón o en ropa de trabajo masculina (Wikipedia, 2013). Se utilizaron hasta ser desplazados casi totalmente por los broches y remaches a presión, que comenzaron a usarse hace una cincuentena de años.

Finalmente la pieza 6 es un fleje o varilla de acero al carbono que presenta una sección uniforme, producto semielaborado de acería de estructura normalizada ferritico-perlitica. (Calvo Rodes, 1963:59-61). Sus lados largos son planos mientras que los cortos son redondeados lo que indica que ha sido obtenida por laminación a partir de una barra de sección circular sección. La estructura no ha sufrido variaciones notables en los últimos doscientos años. La deformación que presenta en un extremo indica que fue golpeado reiteradamente para clavarlo en el terreno a modo de puntal o mojón. 


\section{Consideraciones finales}

El área en cuestión sólo puede haber estado habitada con posterioridad a la denominada "campaña del desierto" es decir a partir de 1870. La tecnología de elaboración y características del conjunto de la muestra ( cuchillos procedentes de un juego fabricado industrialmente en serie, pieza de cobre de un artefacto de combustible líquido con soldadura, varilla obtenida de un redondo por laminación y no por trefilado, presilla fabricada a máquina en serie, partiendo de alambre y de uso normal en ropa de confección son todos indicadores de un período temporal de ocupación para el sitio en cuestión que va de las últimas décadas del siglo XIX a las primeras del XX.

\section{Referencias}

CALVO RODES, R. (1963). Prontuario Metalotécnico. Madrid: INTA.

FONDAZIONE NERI MUSEO ITALIANO DELLA GHISA, (2013). L'illuminazione attraverso $i$ tempi: dalle origini all'utilizzo ottocentesco del gas. Recuperado de http://www.museoitalianoghisa. org/ assets/images/pdf/Storia-Illuminazione-IT.pdf. Consultado 10 octubre 2013.

GILLMAN F. 1886. Enciclopedia Popular Ilustrada de Ciencias y Artes. Madrid: Gras y Cia.

PIFFERETTI A. A. y MARTIGNONI C. L. (2004). Estudio y catalogación de materiales metálicos de Santa Fe La Vieja. Bechis, M. (Comp.) Terceras Jornadas de Arqueología Histórica y de Contacto del Centro Oeste de la Argentina y Seminario de Etnohistoria. Vol 1. Río Cuarto: Universidad Nacional de Río Cuarto: 55-66.

ROLDAN, R. (1959). Antonio Quinquet (1745-1803) en Descubrimientos, inventos y adelantos científicos. Boletín de la Sociedad Española de Historia de la Farmacia (39). 237-240.

SÁNCHEZ BELTRÁN, M. J. (1993). Tijeras de oficios y despabiladeras. Separata de Galería Antiquaria (107). 68-75.

SANCHIS, J. M. (2017). Lámparas de mina españolas. MTIEDIT. Recuperado de http://www. museosm.com/exposiciones/lamparastempestad.pdf.

SCHÁVELZON, D. (1991). Arqueología Histórica de Buenos Aires I. La cultura material porteña de los siglos XVIII y XIX. Buenos Aires: Corregidor.

SHERMAN, M. (2012). The Central Draft Burner: Ami Argand's Contribution to the American Home. Recuperado de http://editors.eol.org/eoearth/wiki/

WIKIPEDIA (2013). Recuperado de https://es.wikipedia.org/wiki/Corchete_(textil).

Recibido: 21/05/2017

Aceptado: 08/09/2017 